\title{
Die Rolle von Viren bei tiefen Atemwegsinfektionen des Erwachsenen ${ }^{1}$ Teil 1: Erreger, Pathogenese und Diagnostik
}

\author{
The Impact of Viruses in Lower Respiratory Tract Infections of the Adult \\ Part I: Pathogenesis, Viruses, and Diagnostics
}

Autoren

Institute
S. R. Ott ${ }^{1,2^{*}}$, P. M. Lepper ${ }^{2^{*}}$, B. Hauptmeier ${ }^{3^{*}}$, R. Bals ${ }^{4^{*}}$, M. W. R. Pletz ${ }^{5^{*}}$, C. Schumann ${ }^{6^{*}}$, C. Steininger ${ }^{7^{*}}$, M. Kleines ${ }^{8^{*}}, \mathrm{H}$. Geerdes-Fenge ${ }^{9^{*}}$

Die Institutsangaben sind am Ende des Beitrags gelistet. eingereicht 12.5.2009 akzeptiert nach Revision 26. 8. 2009

\section{Bibliografie}

DOI http://dx.doi.org/ 10.1055/s-0029-1215232

Online-Publikation: 3. 11. 2009

Pneumologie 2009; 63:

709-717 @ Georg Thieme

Verlag KG Stuttgart · New York ISSN 0934-8387

\section{Korrespondenzadresse}

\section{Dr. Sebastian R. Ott}

Universitätsklinik für

Pneumologie

Universitätsspital Bern

(Inselspital)

3010 Bern

Schweiz

sebastian.ott@insel.ch

Serienherausgeber

B. Schaaf, Dortmund

G. Rohde, Bochum

\section{Zusammenfassung \\ $\nabla$}

Infektionen des Respirationstrakts sind in industrialisierten Ländern eine der häufigsten Ursachen für ärztliche Konsultationen, wobei rund ein Drittel dieser Erkrankungen die tiefen Atemwege betreffen. Hierzu zählen neben der akuten Bronchitis auch die akute Exazerbation einer chronisch obstruktiven Lungenerkrankung (COPD), die ambulant oder nosokomial erworbene Pneumonie und als besondere Entität die Influenza, die sämtliche Abschnitte des Respirationstrakts betreffen kann. Über lange Zeit wurde durch Mangel an geeigneten Untersuchungsmethoden die Bedeutung von respiratorischen Viren bei der Genese dieser Infektionen wahrscheinlich unterschätzt. Da bislang oft eine bakterielle Genese vermutet wurde, erfolgte in vielen Fällen im klinischen Alltag, insbesondere bei der akuten Bronchitis oder der exazerbierten COPD, eine antibiotische Behandlung, die wahrscheinlich nicht erforderlich ist und zudem das Risiko einer Resistenzentwicklung beinhaltet. Erst mit der Einführung von sensitiveren Nachweisverfahren für Viren, wie zum Beispiel der Polymerasekettenreaktion (PCR), gelingt ein sicherer und zuverlässiger Nachweis von respiratorischen Viren, der Aussagen über deren Bedeutung bei Infektionen des tiefen Respirationstrakts erlaubt. Im Rahmen dieser dreiteiligen Serie soll deshalb anhand der aktuellen Literatur ein Überblick über den derzeitigen Kenntnisstand zur Bedeutung von respiratorischen Viren bei tiefen Atemwegsinfektionen des Erwachsenen gegeben werden. Der erste Teil der Serie befasst sich mit den ursächlichen Erregern, der Pathogenese und Diagnostik bei tiefen Atemwegsinfektionen des Erwachsenen. In den folgenden Abschnitten werden Krankheitsbilder (Teil II: akute Bronchitis, exazerbierte COPD, Pneumonie und Influenza) sowie Therapie und Prävention (Teil III) vorgestellt.

${ }^{1}$ Gefördert durch die Paul Ehrlich Gesellschaft (PEG)

\section{Abstract \\ $\nabla$}

In industrialised countries respiratory tract infections are one of the most common reasons for medical consultations. It is assumed that almost one third of these infections include the lower respiratory tract (LRTI), e.g. acute bronchitis, acute exacerbation of chronic obstructive pulmonary disease (COPD), community- or hospital-acquired pneumonia and influenza. Due to a lack of sufficient and valid investigations on the epidemiology of respiratory viruses, their impact on the pathogenesis of LRTI has probably been underestimated for a long time. Therefore, there might have been many cases of needless antibiotic treatment, particularly in cases of acute bronchitis or acute exacerbations of COPD, because of an assumed bacteriological aetiology. Following the introduction of diagnostic procedures with increased sensitivity, such as polymerase chain reaction, it is possible to reliably detect respiratory viruses and to illuminate their role in the pathogenesis of LRTI of the adult. We have reviewed the current literature to elucidate the role of viruses in the pathogenesis of LRTI. The first part of this series deals with the relevant pathogens, pathogenesis, and diagnostic procedures. In the subsequent 2 parts of this series a review will be given on the most common variants of viral LRTI (part II), and therapeutic and preventive options (part III). 


\section{Einleitung}

Infektionen des Respirationstrakts sind in industrialisierten Ländern eine der häufigsten Ursachen von Hausarztkonsultationen [1]. Es ist davon auszugehen, dass rund ein Drittel dieser Infektionen den unteren Respirationstrakt (Lower Respiratory Tract Infection; LRTI) betreffen [2]. Hierzu zählen neben der „akuten Bronchitis“ auch die akute Exazerbation einer chronisch obstruktiven Atemwegserkrankung (Chronic Obstructive Pulmonary Disease; COPD; Acute Exacerbated-COPD; AE-COPD), die ambulant erworbene Pneumonie (Community-acquired Pneumonia; CAP) sowie die Influenza. In der Literatur finden sich Inzidenzraten von 44-50 pro 1000 Einwohner [3,4]. Obwohl der klinische Nutzen einer Antibiotikabehandlung bei bestimmten Formen der LRTI, besonders der akuten Bronchitis, oder Fällen einer AE-COPD wenig belegt ist $[2,5,6]$, werden in Europa in der ambulanten Patientenversorgung die meisten dieser Patienten antibiotisch behandelt [2]. Dieser Tatsache kommt vor dem Hintergrund, dass ein derartig unkritischer Gebrauch von Antibiotika mit einer Zunahme der Resistenzraten einhergeht [7], eine besondere Bedeutung zu.

Über die Ätiologie von LRTI, insbesondere bei leichten und mittelschweren Fällen und somit in der ambulanten Patientenversorgung, ist bislang wenig bekannt. Aber auch bei schwereren Fällen, die eine stationäre Behandlung erfordern (z.B. CAP), gelingt eine Erregerdiagnostik nur bei 30-70\% der Patienten [8,9]. Ursächlich hierfür sind Unterschiede in der jeweils untersuchten Patientenpopulation, der Wahl der verwendeten Untersuchungsmaterialien (Rachenabstrich, Sputum, Nasen- und/oder Rachenspülung, Trachealaspirat, Bronchialsekret, BAL, Blut etc.) sowie Art und Umfang der verwendeten Untersuchungsmethoden (Serologie, Allgemeinkultur, Viruskultur, Polymerase-Kettenreaktion [Polymerase Chain Reaction; PCR] etc.).

Während die ätiologische Bedeutung von Bakterien bei LRTI, insbesondere in der Genese der CAP, durch zahlreiche Studien gut untersucht ist $[8,9]$ und sich diese Kenntnisse auch in den Leitlinien zur Behandlung dieser Infektionen widerspiegeln [10], ist über die Rolle von viralen Atemwegsinfektionen bislang wenig bekannt. Erst in den letzten Jahren ist durch den Einsatz von sensitiveren Untersuchungsmethoden wie der PCR im Rahmen von Studien zur Ätiologie von tiefen Atemwegsinfekten sowie im klinischen Alltag die Rolle von Viren in der Genese von LRTI vermehrt in den Mittelpunkt des Interesses gelangt. So finden sich in neueren Studien je nach Krankheitsbild in bis zu 63\% der Fälle mit Erregernachweis Viren als Ursache [4,8,9,11-13]. Somit wurde die Bedeutung von Viren bei tiefen Infektionen des Respirationstrakts bei Erwachsenen bislang wahrscheinlich unterschätzt.

Vor diesem Hintergrund hat die Arbeitsgruppe „Pneumologie“ der Paul-Ehrlich-Gesellschaft beschlossen, ein Statuspapier zu erstellen, das zum Ziel hat, die Bedeutung von Virusinfekten bei tiefen Atemwegsinfektionen des Erwachsenen zu beleuchten. Anhand der aktuellen Literatur soll neben dem derzeitigen Kenntnisstand zur ätiologischen Bedeutung von Viren bei tiefen Atemwegsinfektionen auch eine allgemeine Übersicht über virale Atemwegsinfektionen gegeben werden. Hierdurch könnten sich möglicherweise Konsequenzen für den klinischen Umgang mit tiefen Atemwegsinfektionen, insbesondere eine Empfehlung zum Einsatz von Antibiotika und/oder antiviralen Medikamenten, ergeben. Ferner ist beabsichtigt, noch offene Fragen bezüglich der Rolle von Viren bei Atemwegsinfektionen, die Ansatz- punkte für künftige Studien geben könnten, zu identifizieren. Diese Übersichtsarbeit stellt jedoch keine Leitlinie dar.

Im ersten Teil der Serie werden die Pathogenese, häufig gefundene Viren und die Diagnostik von viralen Atemwegsinfekten dargestellt. Der zweite Teil wird sich mit den vier klinisch relevanten Formen der viral ausgelösten LRTI (akute Bronchitis, exazerbierte COPD, Pneumonie und Influenza) beschäftigen, der dritte und letzte Teil gibt eine Übersicht über Therapie und Prävention.

\section{Pathogenese von Virusinfekten}

$\nabla$

Für das Überleben von Vertebraten ist es essenziell, über eine Strategie für die Erkennung von „selbst“ und „nicht-selbst“ zu verfügen. Bislang unterscheiden wir innerhalb dieser Strategie „angeborene“ und „erworbene“ Immunität. Der Begriff „erworben“ bezeichnet eine spezielle Form der Immunität, welche wesentlich durch die Umwelt beeinflusst wird, d.h. dadurch, dass der Organismus einem Erreger ausgesetzt war, kommt es zu einer „klonalen Expansion“ bestimmter Zellen, welche schlussendlich ein spezifisches Ziel bekämpfen. Der Begriff „angeboren“ beschreibt alle anderen Formen.

\section{Infektion der Wirtszelle}

Viren können ohne eine Wirtszelle nicht „überleben“. Zur Replikation schleusen sie ihr Genom, bestehend aus einzel- oder doppelsträngiger RNA oder DNA, in die Wirtszelle ein. Obgleich es signifikante Unterschiede zwischen den Mechanismen der Infektion einzelner Zellen gibt, bestehen doch, da alle Viren vor ähnlichen Problemen auf dem Weg von Wirtszelle zu Wirtszelle stehen (z.B. pH der Umgebung, Temperatur, Proteasen), Gemeinsamkeiten bei der Vielzahl bisher untersuchter Viren. Für zahlreiche Viren ist die Lösung dieser Probleme eine Verbindung zwischen den letzten Schritten der Virusvermehrung und der Infektion ( $\bullet$ Abb. 1). Somit ist das Virion eine Zwischenform im Lebenszyklus des Virus. Die letzten Stadien der Virusvermehrung stellen häufig proteolytische Veränderungen von Strukturproteinen dar (in der Regel Oberflächenglykoproteine im Falle behüllter Viren oder Kapsidproteine im Falle unbehüllter Viren). Diese führen zu einem sogenannten metastabilen Zustand, der es dem Virus aufgrund bestimmter energetischer Barrieren erst dann erlaubt, in die Zielzelle einzudringen, wenn der metastabile $\mathrm{Zu}$ stand durch einen passenden Trigger (Rezeptorbindung oder endosomale pH-Änderung oder beides) aufgehoben wird. Dann können hydrophobe Molekülreste freigelegt werden und zur Anheftung an die Zellmembran führen. Bei behüllten Viren führt die Anheftung zu einer Fusion der Hülle mit der Zellmembran, bei unbekapselten Viren müssen die hydrophoben Reste eine Pore bilden, damit das Virus eindringen kann. Ferner sind sowohl bei behüllten als auch unbehüllten Viren Endozytosevorgänge von Bedeutung. Grundsätzlich kann die Infektion der Zelle also auf zwei Wegen stattfinden, einem pH-getriggerten und einem rezeptorgetriggerten.

\section{Toll-like Rezeptoren}

Der Erkennungsprozess der angeborenen Immunität erfolgt über Rezeptoren, die in der Keimbahn kodiert sind, wodurch ihre Spezifität genetisch starr festgelegt ist. Dies ist von Vorteil, da diese Rezeptoren, deren Spezifität sich unter dem Selektionsdruck der Evolution herausgebildet hat, nun von Generation zu Generation weiter gegeben werden. Sie werden Mustererkennungsrezeptoren (Pattern recognition receptor; PRR) genannt und erkennen 


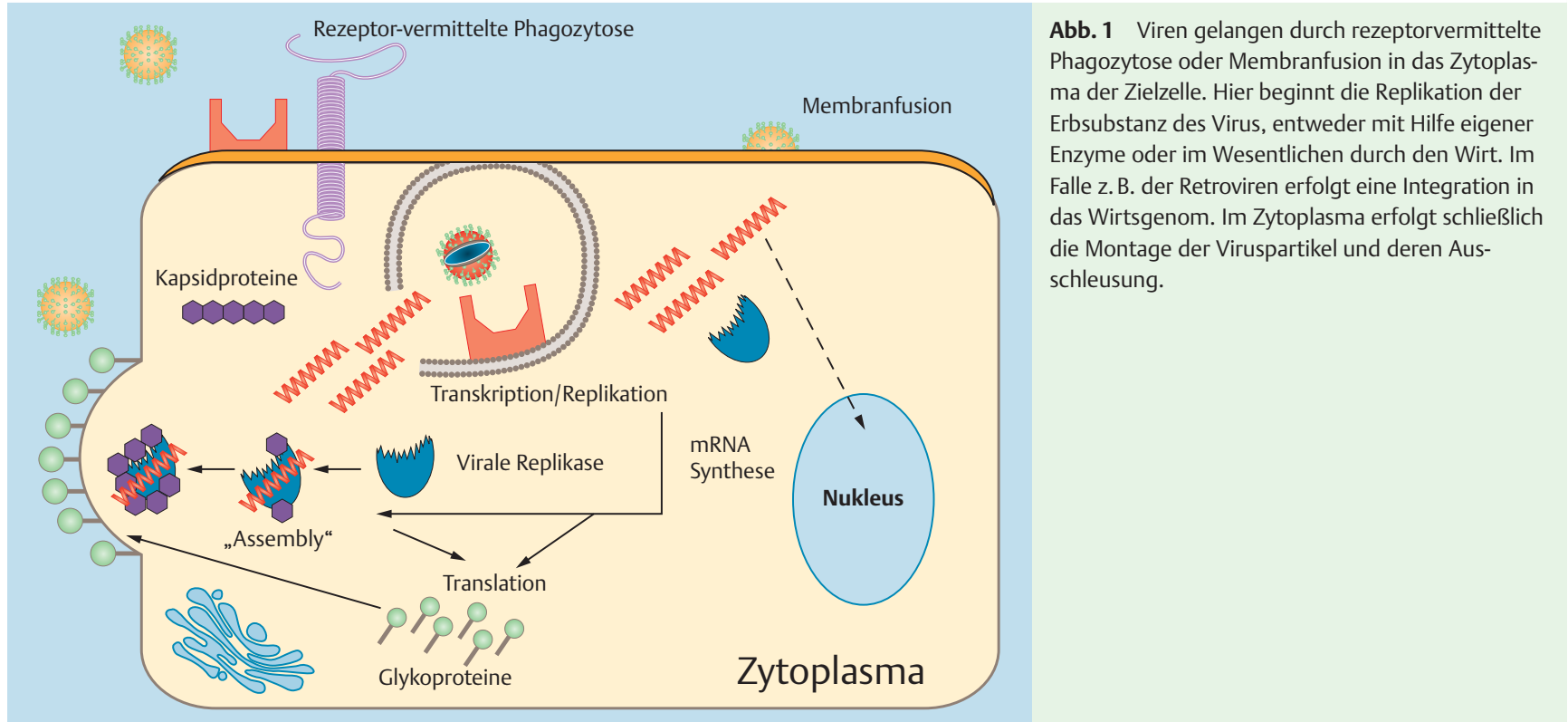

evolutiv hoch-konservierte Strukturen, die vielen Mikroorganismen einer Art gemeinsam sind. Diese Strukturen zeichnen sich insbesondere dadurch aus, dass sie vorzugsweise nicht im Wirtsorganismus zu finden sind.

Toll, ein transmembranes Protein hat eine entscheidende Funktion für die angeborene Immunität als PRR [14]. Humane Tolllike Rezeptoren (TLR) sind Schlüsselproteine, welche uns die Erkennung von Infektionen ermöglichen. Innerhalb der Zelle existieren z.T. ähnliche Strukturen (z.B. mRNA). Um nun „selbst“ von „nicht-selbst“ unterscheiden zu können, überwachen TLRs sowohl den extrazellulären Raum wie auch das Zytosol und endosomale Kompartimente. Während der Replikation der Viren innerhalb der Wirtszelle entstehen verschiedene Nukleinsäurederivate mit „viraler Signatur“. Sie aktivieren über TLRs durch konservierte strukturelle Motive („Signatur“) die angeborene und schließlich auch die adaptive Immunität.

Viren waren die letzten Pathogene, deren Erkennung durch TLRs bestätigt werden konnte [15-20]. Sie werden im Wesentlichen von den TLRs erkannt, die spezialisiert auf die Erkennung von unmethylierter DNA (TLR9), dsRNA (TLR3) und ssRNA (TLR7 und 8) sind. Es ist eine Herausforderung zu entscheiden, ob Nukleinsäuren „selbst“ oder „nicht-selbst“ sind, da die Unterschiede sehr subtil sind. So kommt zum Beispiel einzelsträngige RNA (ssRNA) sehr häufig in eukaryotischen Zellen vor, aber auch doppelsträngige RNA (dsRNA) ist bisweilen vertreten. Ferner sind auch CpGDinukleotide im humanen Genom unmethyliert und sollten daher von TLR9 erkannt werden können. TLR3, 7, 8 und 9 erkennen strukturelle Differenzen zwischen viralen und eukaryotischen Nukleinsäuren, aber es ist vermutlich zwingend erforderlich, dass die Rezeptoren ihre Liganden im endosomalen Kompartiment registrieren [21,22] Dies ist ein Kompartiment, das unter normalen Umständen frei von Wirts-RNA oder -DNA ist [23].

Aber nicht nur virale Nukleinsäuren, auch virale Proteine können durch TLRs erkannt werden [17]. Haben Viren das Zytosol erreicht, entziehen sie sich zunächst der Erkennung durch TLRs. Dann können virale Bestandteile intrazellulär durch sog. RNAHelicasen auch TLR-unabhängig wahrgenommen werden $[24,25]$. Wie erst kürzlich gezeigt wurde, erkennen diese Helicasen virale ssRNA mit 3 Phosphatresten am 5'-Ende [26,27].
Alle Zellen an exponierter Stelle tragen PRR. Entsprechend gilt dies für Pneumozyten [22]. Das bedeutet aber auch, dass das Epithel der Atemwege über Rezeptoren verfügt, die es prinzipiell anfällig für virale Infektionen macht. Aus dem oben Gesagten geht auch hervor, dass andere Zellen, z. B. Kardiomyozyten, durch respiratorische Erreger infizierbar sind [28].

\section{Induktion einer antiviralen Antwort}

Nach Aktivierung eines PRR wird ein Signal an den Zellkern weitergeleitet und die Transkription spezifischer Genabschnitte eingeleitet. Nach Ligation von TLR3, 7, 8 und/oder 9 kommt es zu einer Ausschüttung von Typ-I-Interferon (IFN $\alpha$ und $\beta$ ). Typ-IInterferone (IFN) spielen eine bedeutende Rolle für den Start eines antiviralen Programms. IFN-induzierbare Gene beeinflussen u.a. Proteinbiosynthese, Zellzyklus und Apoptose. Darüber hinaus stimulieren Typ-I-IFN die Reifung von dendritischen Zellen (DCs), verstärken die Zytotoxizität von natürlichen Killerzellen und induzieren die Differenzierung Virus-spezifischer zytotoxischer T-Zellen und verbinden so angeborene und adaptive Immunität $[29,30]$.

\section{Antimikrobielle Peptide}

Als Effektormechanismen gegen virale Infektionen stehen verschiedene Strategien zur Verfügung. Eine wichtige Funktion haben Natural-killer(NK)-Zellen [31]. Diese Zellen machen ungefähr 5-20\% der Lymphozyten vieler Organsysteme aus und sind auf die Erkennung und Elimination pathogen-infizierter Zellen und Tumorzellen spezialisiert. Diese Aktivität wird über die Abgabe löslicher Mediatoren und Zytolyse vermittelt.

Antimikrobielle Peptide sind Effektormoleküle der angeborenen Immunität mit direkter antimikrobieller Wirkung und einer Reihe von Funktionen als Mediatoren. Defensine und Cathelizidine sind prototypische Peptidfamilien, die beim Menschen gefunden wurden. Der Wirkungsmechanismus dieser endogenen Antibiotika beruht in den meisten Fällen auf Interaktionen mit Biomembranen. Die Expression einiger antimikrobieller Peptide wird durch Virusinfektion induziert, gleichzeitig haben Peptide auch Wirkung gegenüber Viren [32]. So hemmen neutrophile alphaDefensine HSV-1, HVS-2 und in geringerem Maß auch CMV. Diese Peptide besitzen auch eine Aktivität gegen HIV. 
Im Folgenden soll am Beispiel der akuten viralen Exazerbation der chronisch obstruktiven Lungenerkrankung (COPD) exemplarisch die Pathogenese viraler tiefer Atemwegsinfektionen dargestellt werden.

Respiratorische Viren, z.B. Rhinoviren, die als einer der häufigsten viralen Erreger einer Exazerbation identifiziert und bislang am besten untersucht sind, binden über interzelluläre Adhäsionsmoleküle (z.B. ICAM-1) an das Atemwegsepithel und können es somit direkt durch einen Verlust von Zilien tragenden Zellen schädigen. Ferner verursachen sie eine erhöhte Plasmaexsudation sowie vermehrte Schleimproduktion [33]. Durch Bindung an ICAM-1 wird die Bildung von weiteren ICAM-1-Molekülen induziert [34], was eine chemotaktische Wirkung auf neutrophile Granulozyten hat. Dadurch kommt es im Rahmen einer Exazerbation zu einer Zunahme der bereits im stabilen Zustand vorhandenen Entzündungsreaktion. So wurden in einigen Studien erhöhte Werte für TNF- $\alpha$, IL-8 [35,36], LeukotrienB4 (LTB4) [37], epithelial-derived neutrophil attractant (ENA-78) [36] und Endothelin-1 [38], einem Bronchokonstriktor, beschrieben. Darüber hinaus führen Rhinoviren zur Aktivierung des Transkriptionsfaktors Nuklearer-Faktor-KB (NF-kB) [34], der eine wichtige Rolle in der Regulation verschiedener Zytokine innehat und somit ebenfalls proinflammatorisch wirkt.

\section{Erreger}

Infektionen des unteren Respirationstraktes (LRTI) können durch Viren aus unterschiedlichen Familien ( $\bullet$ Tab. 1 ) ausgelöst werden. Einige sind seit langer Zeit bekannt, andere sind erst in den letzten Jahren neu entdeckt und in ihrer Bedeutung für LRTI wahrgenommen worden. Im Folgenden sollen überwiegend Viren der zweiten Gruppe ausführlicher vorgestellt werden. Auf nicht saisonale Influenza-Erreger wird nicht explizit eingegangen, da sie entweder bisher für Deutschland keine klinische Bedeutung haben (Vogelgrippe, Influenza-A-Virus Subtyp H5N1) oder aufgrund des kurzfristigen Auftretens keine endgültigen Aussagen getroffen werden können (Schweinegrippe InfluenzaA-Virus Subtyp H1N1-swine). Aktuelle Informationen diesbezüglich können den Veröffentlichungen der WHO (www.who.int) und des Robert Koch Instituts (www.rki.de) entnommen werden. Das humane Bocavirus (hBoV), ein Parvovirus, wurde 2005 in Respirationstraktmaterial von Patienten mit respiratorischem Infekt entdeckt. Es ist weltweit verbreitet und befällt Menschen aller Altersgruppen. Hospitalisierungen bei Erwachsenen sind sehr selten. HBoV wurde bei $1,5 \%$ bis $19 \%$ aller Kinder mit Respirationstraktinfekten nachgewiesen, häufig als Koinfektion mit etablierten respiratorischen Viren. HBoV-spezifische Krankheitssymptome konnten bisher nicht identifiziert werden [39].

Humanpathogene Coronaviren (hCoV) sind seit Jahrzehnten bekannt. CoV-OC43 und CoV-229E werden für 10-25\% aller Schnupfenfälle verantwortlich gemacht und können auch Bronchitis und Pneumonie auslösen. In den letzten Jahren wurden weitere Mitglieder dieser Familie entdeckt. Das SARS-CoV wurde 2003 als Ursache für das severe acute respiratory syndrome (SARS) identifiziert [40]. Im Jahr 2004 wurde das weltweit verbreitete CoV-NL63 isoliert [41]. Es befällt bevorzugt chronisch kranke Kinder und konnte in 1\% - 7\% der Atemwegsmaterialien respiratorisch erkrankter Patienten nachgewiesen werden. Eine Assoziation des Virus mit Pseudo-Krupp wird ebenfalls diskutiert. CoV-HKU1 wurde 2005 in Hongkong entdeckt und verursacht Erkrankungen der oberen und unteren Atemwege. Das
Tab. 1 Viren mit Bedeutung bei tiefen Atemwegsinfekten; ds-DNA: Doppelstrang Desoxyribonukleinsäure; ss-RNA: Einzelstrang Ribonukleinsäure.

\begin{tabular}{|lll}
\hline Adenoviridae & humanes Adenovirus & ds-DNA \\
\hline Coronaviridae & humanes Coronavirus & ss-RNA \\
& - CoV-OC43 & \\
& - CoV-229E & \\
& - SARS-CoV & \\
& - CoV-NL63 & \\
\hline Humane Herpesviridae & Cytomegalievirus & \\
& Herpes simplex 1 und 2 & ds-DNA \\
& Varizella-Zoster-Virus & ds-DNA \\
\hline Orthomyxoviridae & Influenzavirus A & ss-RNA \\
\hline Parvoviridae & Influenzavirus B & ss-RNA \\
\hline Picornaviridae & humanes Bocavirus & ss-DNA \\
\hline Paramyxoviridae & humanes Rhinovirus & ss-RNA \\
& Coxsackievirus A und B & ss-RNA \\
& Echoviren & ss-RNA \\
\hline & Respiratory Syncytial Virus & ss-RNA \\
& Parainfluenzavirus & ss-RNA \\
& humanes Metapneumovirus & ss-RNA \\
& Masernvirus & ss-RNA
\end{tabular}

Virus wurde bei $1 \%$ - $19 \%$ respiratorisch erkrankter Personen nachgewiesen [42]. Wie die meisten der in den letzten Jahren neu entdeckten respiratorischen Viren wird CoV-HKU1 vermehrt bei Kindern mit Grunderkrankungen nachgewiesen.

Das humane Metapneumovirus (hMPV), ein Paramyxovirus, wurde 2001 entdeckt [43]. Es ist weltweit verbreitet und verursacht bei einer Inzidenz von 1,5\% bis 25\% leichte wie auch schwere Infektionen des oberen und unteren Respirationstraktes bei Säuglingen und Kleinkindern [44]. Das hMPV trägt signifikant zur Morbidität und Mortalität von Frühgeborenen sowie von Kleinkindern mit Grunderkrankungen bei. Es wird auch als Trigger für Asthma diskutiert. Bei Erwachsenen sind symptomatische Erkrankungen seltener (hMPV-Nachweis bei bis zu 4\% von Erwachsenen mit ambulant erworbener Pneumonie) und betreffen zumeist immunkompromittierte Patienten oder solche mit chronisch obstruktiven Lungenerkrankungen [45].

Humane Herpesviren spielen als Erreger von Respirationstrakterkrankungen bei immunkompromittierten Menschen eine Rolle [46 - 48]. Das humane Cytomegalievirus (CMV) muss bei Organtransplantierten, HIV-Infizierten und anderen Menschen mit bekannter Immunsuppression im Fall einer respiratorischen Symptomatik immer als Differenzialdiagnose berücksichtigt und diagnostisch mit erfasst werden. Auch Herpes-Simplex-Virus (HSV), Varizella-Zoster-Virus (VZV) und Epstein-Barr-Virus (EBV) sollten aber bei atypischen Krankheitsverläufen mit berücksichtigt werden.

Humane Rhinoviren (hRV) gehören zur Familie der Picornaviren, sind seit Jahrzehnten bekannt und weltweit verbreitet. Mehr als 100 Rhinovirustypen können unterschieden werden, die nach Infektion keine nachhaltige Immunität induzieren. Rhinoviren sind eine wichtige Ursache von Infektionen der oberen Atemwege und werden meist mit banalen Erkältungen in Zusammenhang gebracht. Zunehmend zeigt sich, dass sie auch für Infektionen der unteren Atemwege von Bedeutung sind $[49,50]$. Ihre Assoziation mit Tracheobronchitis ist seit langer Zeit etabliert. Von acht Probanden ohne Immunität gegen Rhinoviren, die ein rhinovirushaltiges Aerosol inhalierten, entwickelten alle Rhinitis, Husten und Allgemeinsymptome, drei davon mit Zeichen einer schweren akuten Tracheobronchitis, drei mit mäßiger Tracheobronchitis und zwei mit überwiegend Schnupfen mit nur mäßigem Husten [51]. 


\begin{tabular}{|c|c|c|c|c|c|}
\hline Virus/Testverfahren & $\begin{array}{l}\text { Schnell- } \\
\text { test }\end{array}$ & PCR & $\begin{array}{l}\text { Antigen-Immun- } \\
\text { fluoreszenztest }\end{array}$ & $\begin{array}{l}\text { Antigen- } \\
\text { ELISA }\end{array}$ & $\begin{array}{l}\text { Zell- } \\
\text { kultur }\end{array}$ \\
\hline Adenoviren & - & + & + & - & + \\
\hline Bocavirus & - & + & - & - & + \\
\hline \multicolumn{6}{|l|}{ Coronaviren } \\
\hline - CoV-229E & - & + & + & - & + \\
\hline - CoV-HKU1 & - & + & - & - & - \\
\hline - CoV-NL63 & - & + & - & - & + \\
\hline - CoV-OC43 & - & + & + & - & + \\
\hline - SARS-CoV & - & + & + & + & + \\
\hline $\begin{array}{l}\text { Enteroviren } \\
\text { (Coxsackie- und Echoviren) }\end{array}$ & - & + & - & - & + \\
\hline \multicolumn{6}{|l|}{ Herpesviren } \\
\hline$-\mathrm{CMV}$ & - & + & + & - & + \\
\hline$-E B V$ & + & + & & - & + \\
\hline - HSV & - & + & + & - & + \\
\hline - VZV & - & + & + & - & + \\
\hline Influenzavirus A/B & + & + & + & + & + \\
\hline \multicolumn{6}{|l|}{ Paramyxoviren } \\
\hline - Metapneumovirus & - & + & + & + & + \\
\hline - Parainfluenzaviren & - & + & + & - & + \\
\hline - RSV & + & + & + & - & + \\
\hline - Rhinoviren & - & + & + & + & + \\
\hline
\end{tabular}

Tab. 2 Etablierte Nachweisverfahren wichtiger viraler Respirationstraktpathogene (nicht alle genannten Testverfahren sind in Routinediagnostiklabors verfügbar).

\section{Diagnostik}

\section{$\nabla$}

\section{Untersuchungsmaterial}

Für alle In-vitro-Diagnostikverfahren ist die Präanalytik von großer Bedeutung. $\mathrm{Zu}$ lange oder falsche Lagerung mindert die Qualität des Patientenmaterials und führt somit zu schlechteren Testergebnissen. Viren zeigen eine geringe Umweltstabilität (Tenazität) außerhalb von feuchtem Milieu. So überleben Influenzaviren auf inerten Oberflächen nur wenige Stunden, können aber bei $4{ }^{\circ} \mathrm{C}$ bis zu 38 Tage im Stuhl nachgewiesen werden. Die Tenazität unbehüllter Viren ist größer als die behüllter Viren.

Für den Direktnachweis respiratorischer Viren geeignete Materialien sind bronchoalveoläre Lavage (BAL), Rachenspülwasser, Trachealsekret, tiefe Nasen-/Rachenabstriche und Mukusfallen bei Kindern. Aufgrund der nicht standardisierten Gewinnungsmethoden ist die Eignung des Materials stark von den Entnahmemodalitäten abhängig. Die Verwendbarkeit von induziertem Sputum, einem nicht-invasiven Verfahren zur Gewinnung von Atemwegssekreten, ist nicht systematisch hinsichtlich der Verwendbarkeit für die beschriebenen Methoden evaluiert worden. Studien, die induziertes Sputum verwenden, weisen jedoch darauf hin, dass auch hier verschiedene Methoden zur Identifikation von viralen Erregern angewandt werden können [52 - 55]. Serum kann zur Bestimmung virusspezifischer Antikörpertiter und verschiedener Akutphaseproteine (z.B. C-Reaktives Protein, Procalcitonin, Serum Amyloid A), die möglicherweise eine Differenzierung zwischen bakteriellen und viralen Infekten erlauben, verwendet werden. Ein noch in der experimentellen Phase befindliches Untersuchungsmaterial ist das Atemwegskondensat.

\section{Virusnachweis}

Eine detaillierte Übersicht zum Nachweis von Atemwegsviren wurde im Rahmen der Serie „Diagnostik pulmonaler Infektionskrankheiten“ in der Januar-Ausgabe dieser Zeitschrift veröffentlicht (Rohde et al., Pneumologie 2009; 63 : 14 - 22) [56]. Zur Diagnose viraler LRTI wurden zahlreiche Nachweisverfahren für virusspezifische Antikörper oder zum Virusdirektnachweis etabliert. Antikörper sind zu Beginn der symptomatischen Krank- heitsphase meist noch nicht nachweisbar. Aus diesem Grund ist der Antikörpernachweis durch Komplement-Bindungsreaktion (KBR), Enzyme Linked Immunosorbent Assay (ELISA) oder indirekten Immunfluoreszenztest (IFT) zur Identifizierung des Erregers eines akuten viralen Respirationstraktinfektes ungeeignet und nur für retrospektive Betrachtungen und epidemiologische Studien sinnvoll. Viele ältere Studien basieren ausschließlich auf Antikörperbestimmungen. Das mindert ihre Aussagekraft deutlich.

Virusdirektnachweise sind die Methode der Wahl zum Nachweis respiratorischer Viren. Das klassische Verfahren der Virusanzucht mit Zellkulturen ist für die meisten respiratorischen Viren etabliert (siehe Tab. 2), spielt aber aufgrund des methodischen Aufwandes und der langen Verfahrensdauer keine Rolle in der Akutdiagnostik.

Die Anforderungen an methodischen Aufwand und Zeitnähe des Testergebnisses werden von Schnelltesten (z.B. Immunchromatografie), Polymerasekettenreaktion (PCR), Antigen-IFT und Antigen-ELISA erfüllt ( Tab. 3). Der zeitliche Vorteil bei der Anwendung der Schnellteste (weniger als 30 Minuten) gegenüber den anderen Verfahren (IFT: 90 Minuten, real time PCR: 120 Minuten, ELISA: 240 Minuten) fällt im Routinelabor aufgrund der Zeiten für Materialtransport und Befundübermittlung nicht ins Gewicht. Mit allen Verfahren ist eine Befundung am Tag der Einsendung möglich. Entscheidend für die Beurteilung der Testverfah-

Tab. 3 Sensitivität unterschiedlicher Direktnachweisverfahren für Influenzaviren.

\begin{tabular}{|lll|}
\hline $\begin{array}{l}\text { Testverfahren } \\
\text { Schnellteste }\end{array}$ & $\begin{array}{l}\text { Sensitivität } \\
63 \%-85 \%\end{array}$ & $\begin{array}{l}\text { Quelle } \\
\text { Agoritsas et al, 2006 [79] } \\
\text { Grijalva et al., 2007 [80] }\end{array}$ \\
\hline PCR & $92,3 \%$ & $\begin{array}{l}\text { Carraro et al., 2007 [81] } \\
\text { Steininger et al. } 2002 \text { [57] }\end{array}$ \\
\hline $\begin{array}{l}\text { Antigen-Immun- } \\
\text { fluoreszenztest }\end{array}$ & $84 \%-93,6 \%$ & $\begin{array}{l}\text { Carraro et al., 2007 [81] } \\
\text { Antigen-ELISA }\end{array}$ \\
\hline
\end{tabular}


ren ist somit die Sensitivität. Hier kann der IFT der PCR gleichwertig sein; Schnellteste und Antigen-ELISAs fallen deutlich dahinter zurück ( Tab. 3) $[57,58]$. Der IFT ist schlechter automatisierbar als die PCR und stellt höhere Anforderungen an die Materialqualität. Etwa 5\% der eingesendeten Materialien sind nicht für den IFT geeignet, wohl aber für die PCR [59]. Demgegenüber stehen die höheren Kosten der PCR. In $\bullet$ Tab. $\mathbf{2}$ ist die Verfügbarkeit der genannten Verfahren zum Nachweis wichtiger viraler Respirationstrakterreger zusammengefasst.

\section{Akut-Phase-Proteine}

Akut-Phase-Proteine (C-reaktives Protein [CRP], Procalcitonin [PCT], Haptoglobin, etc.) werden im klinischen Alltag als Indikatoren für akute entzündliche Prozesse verwendet. Diese Proteine sind endogene Entzündungsmediatoren, die meist von Hepatozyten als Antwort auf akute und chronische Entzündungen, einschließlich Infektionen, Traumata und unterschiedlicher Neoplasien produziert werden [60]. Obwohl der Nachweis dieser Parameter sehr unspezifisch ist, sind gemessene Serumspiegel von großem Nutzen, da sie Vorhandensein und Intensität entzündlicher Prozesse anzeigen können. Plasmaspiegel von CRP werden sehr häufig für klinische Entscheidungen herangezogen, sind jedoch von begrenztem Nutzen bei Patienten mit akuten viralen Infektionen, da CRP-Werte bei diesen Patienten meistens normwertig oder nur gering erhöht sind [61].

\section{Procalcitonin und Serum Amyloid A}

Procalcitonin (PCT) ist ein Propeptid des Calcitonins, das unter physiologischen Bedingungen in den C-Zellen der Schilddrüse gebildet wird. Obwohl für PCT bislang keine eigene hormonale Aktivität bekannt ist, gilt es als ein Marker zur Diagnostik von schweren bakteriellen Infektionen. Im Serum besitzt es eine Halbwertzeit von ca. 25-30 Stunden und der Nachweis erfolgt in der Regel aus dem Serum mit Hilfe eines immunoluminometrischen Assays. Es steht auch ein Schnelltest (Bedside-Test, z. B. PCT- $Q^{\circledR}$ der Fa. B.R.A.H.M.S., Berlin) zur Verfügung, der nach ca. 30 Minuten ein semiquantitatives Ergebnis liefert [62].

Normalerweise ist PCT beim Gesunden mit den Standardmessmethoden nicht nachweisbar $(<0,1 \mathrm{ng} / \mathrm{ml})$. Es konnte jedoch gezeigt werden, dass die PCT-Konzentratrion im Serum im Rahmen von schweren bakteriellen, parasitären oder fungalen Infekten mit systemischer Manifestation regelhaft deutlich ansteigt (bis $>100 \mathrm{ng} / \mathrm{ml}$ ). Neben den C-Zellen der Schilddrüse als physiologischen Syntheseort konnte im Rahmen von schweren Infekten sowohl in Leukozyten als auch in neuroendokrinen Zellen und dem Lungengewebe eine procalcitoninähnliche Aktivität nachgewiesen werden.

Im Gegensatz zu Zytokinen (z. B. Tumornekrosefaktor- $\alpha$ oder Interleukin-6) oder dem C-reaktiven Protein (CRP), die auch häufig im Zusammenhang mit chronisch-inflammatorischen Erkrankungen wie Lupus erythematodes oder Antikörper-assoziierten Vasculitiden erhöht sind, finden sich PCT-Erhöhungen bei diesen Erkrankungen lediglich, wenn zusätzlich eine infektiöse Komplikation hinzukommt.

Ein Nutzen der PCT-Bestimmung liegt möglicherweise in der Differenzierung zwischen viralen und bakteriellen Infektionen. Bei systemischen Inflammationen durch Viren finden sich keine oder nur moderate PCT-Serumspiegelerhöhungen, und es konnte eine höhere Sensitivität für PCT bei der Unterscheidung von bakteriellen und viralen Infekten im Vergleich zum CRP (92\% vs. $86 \%)$ nachgewiesen werden [61,63]. Darüber hinaus konnte in mehreren prospektiven Studien gezeigt werden, dass es ohne ne- gativen Einfluss auf die Ausheilung zu einer Einsparung von Antibiotika bei LRTI kam, wenn die Indikation zur Verordnung von Antibiotika am PCT-Serumspiegel orientiert wurde [64,65].

Ein Schwachpunkt ist, dass sich deutlich erhöhte PCT-Spiegel lediglich im Rahmen von schweren Infektionen mit systemischer Mitbeteiligung finden. So sinkt die Spezifität für den Nachweis einer schweren Infektion von $72 \%$ bei Patienten mit einer PCTKonzentration $>1,5 \mathrm{ng} / \mathrm{ml}$ auf $35 \%$ bei Patienten mit einem Spiegel $<0,1 \mathrm{ng} / \mathrm{ml}$. Damit liegt die Stärke der PCT-Bestimmung in der Erkennung von Patienten mit septischen Komplikationen im Rahmen von bakteriellen Infekten.

Serum Amyloid A (SAA) gehört zu einer Familie von Apolipoproteinen, die von Hepatozyten als Antwort auf durch Monozyten sezernierte Zytokine produziert werden. Serumspiegel von SAA korrelieren gut mit jenen von CRP, sind jedoch 5- bis 11-fach höher als CRP-Werte bei akuten Virusinfektionen [66,67]. Insbesondere bei akuten respiratorischen Virusinfektionen könnte SAA in Zukunft ein wertvoller Parameter für die Unterscheidung zwischen viraler und bakterieller Genese werden.

\section{Atemkondensat (Exhaled Breath Condensate; EBC)}

Die Gewinnung von EBC erfolgt nicht-invasiv mit sogenannten Kondensatoren. Die Sammlung erfolgt sitzend, dabei atmet der Patient ruhig über ein Mundstück und ein daran angeschlossenes Ein- und Ausatemventil in den Kondensator hinein. Nach etwa 10 min lassen sich ca. $1-3 \mathrm{ml}$ Kondensat gewinnen, das zu >99\% aus Wasserdampf besteht und dessen Ursprung aus allen Teilen des Atmungsapparates stammt [68].

\section{Biomarker im Atemkondensat}

Eine Vielzahl von Biomarkern (>100) u. a. Zytokine (TNF- $\alpha$, IL-1 $\beta$, IL-2, 4, 6, 8 und 10, IFN- $\gamma$ ), Prostanoide und Leukotriene (z.B. $\left.\mathrm{PGE}_{2}, \mathrm{LTB}_{4}\right), 8$-Isoprostan, $\mathrm{NO}$ und abgeleitete Verbindungen $\left(\mathrm{NO}_{2}{ }^{-}, \mathrm{NO}_{3}{ }^{-}\right), \mathrm{H}_{2} \mathrm{O}_{2}$, Ammoniak $\left(\mathrm{NH}_{3}, \mathrm{NH}_{4}{ }^{+}\right)$, aber auch verschiedene Tumormarker, Wachstumsfaktoren wie Erythropoetin und VEGF sowie Endothelin-1 und Adenosin konnten bislang nachgewiesen werden [69].

\section{Atemkondensat als diagnostisches Werkzeug bei Atemwegsinfektionen}

Bei der CAP konnten bislang $\mathrm{NO}_{3}{ }^{-}, \mathrm{H}_{2} \mathrm{O}_{2}$ und Thiobarbituratsäure (TBARs) in erhöhter Konzentration nachgewiesen werden $[69,70]$. Möglicherweise ist der $\mathrm{pH}$ des EBC ein brauchbarer Marker für entzündliche Lungenerkrankungen [71,72]. Der $\mathrm{pH}$ im Atemkondensat ist normalerweise leicht alkalisch ( $\mathrm{pH} \mathrm{7-8),}$ bei entzündlichen Lungenerkrankungen, insbesondere deren Exazerbationen (Asthma, COPD, CF, ARDS), nimmt die Azidität zu [72 - 75]. Hinweise aus Tierexperimenten, dass auch bei viralen Atemwegsinfektionen Marker des oxidativen Stress eine Rolle spielen, finden sich in einer erhöhten Aktivität der SuperoxidDismutase in der bronchoalveolären Lavage bei der Influenza-Infektion der Maus [76]. Für die Diagnostik der viralen Pneumonie könnte der Nachweis von Virus-RNA bzw. -DNA Eingang finden. Systematische Daten dazu fehlen bislang. Der Nachweis humaner DNA im EBC gelang bei Patienten mit Bronchialkarzinom. Mittels PCR konnten Mutationen im p53 Gen identifiziert werden $[77,78]$. 


\section{Nutzen der Atemkondensatmethode in der Diagnostik der LRTI}

Die Atemkondensatmethode hat sich in der Diagnostik und im Monitoring entzündlicher Atemwegsinfektionen als gut reproduzierbare und einfach durchzuführende Methode etabliert. Eine Diskriminierung zwischen bakteriellen und viralen Infektionen ist jedoch noch nicht möglich. Der Nachweis von Virus-RNA oder -DNA im Atemkondensat ist bislang nicht geführt worden, könnte sich jedoch zu einem nützlichen diagnostischen Hilfsmittel entwickeln.

\section{Zusammenfassung}

Eine Vielzahl von verschiedenen Viren kann Infektionen des Respirationstrakts auslösen. Meist gelangen die Viren durch rezeptorvermittelte Phagozytose oder Membranfusion in das Zytoplasma der Wirtszellen. Anschließend findet in ihr die Replikation des viralen Erbguts entweder mit Hilfe eigener Enzyme oder Nutzung der Wirtzelle statt. Abschließend erfolgt die „Montage“ der neuen Viruspartikel und deren Ausschleusung, was häufig zum direkten Untergang der Wirtszelle führt. Ferner werden durch immunkompetente Zellen auch Inflammationsmediatoren freigesetzt, die zur entsprechenden Klinik beitragen können.

Bis vor einigen Jahren erfolgte der Virusnachweis meist indirekt durch serologische Untersuchungen oder durch eine zeitaufwendige Anzucht. Deshalb erfolgte in vielen früheren Studien oftmals keine virologische Diagnostik und, falls sie erfolgte, waren die Ergebnisse aufgrund methodischer Schwächen (z.B. Serologie) nicht sicher aussagekräftig. Dies führte zu einer Vernachlässigung der Rolle von Viren bei der Genese von tiefen Atemwegsinfekten. Erst mit der Entwicklung von sensitiveren Nachweismethoden wie der PCR rückt die wahre Bedeutung von Viren immer mehr in den Fokus des Interesses. So konnten neuere Studien eindrücklich zeigen, dass je nach Krankheitsbild Viren bei mehr als 60\% der Patienten mit Erregernachweis nachgewiesen werden konnten. Interessanterweise wurden nicht nur die bislang schon bekannten Viren wie z. B. Influenzaviren, Parainfluenzaviren, Rhinoviren oder RSV detektiert, sondern es wurden auch neue, bislang unbekannte Viren entdeckt oder Viren gefunden, die nicht typischerweise mit Atemwegsinfekten in Verbindung gebracht wurden, wie z. B. Herpesviren.

Für den direkten Virusnachweis sind zahlreiche Atemwegsmaterialien geeignet. Hierzu zählen neben Rachenspülwasser auch Trachealsekret, tiefe Rachenabstriche und die broncho-alveoläre Lavage (BAL). Wichtig ist, dass zellreiche Proben besser geeignet sind und dass aufgrund der geringen Tenazität der meisten Viren eine zu lange oder falsche Lagerung die Qualität des Patientenmaterials deutlich vermindert. In der Routinediagnostik werden heutzutage meist PCR und IFT verwendet. Für die „Notfalldiagnostik" gerade während Epidemien stehen zum Teil auch Schnellteste zur Verfügung, die jedoch eine deutlich geringere Sensitivität aufweisen. Ein weiteres Patientenmaterial, das eventuell zur Diagnostik bei tiefen Atemwegsinfekten beitragen kann, ist das Atemkondensat (EBC). Ein großer Vorteil ist, dass es im Gegensatz z. B. zur BAL nicht invasiv gewonnen werden muss. Erste Studien zeigen, dass hierdurch auch Aussagen zur Inflammation in den tiefen Atemwegen möglich sind. Leider gibt es bislang noch keine Untersuchungen bezüglich der Einsatzmöglichkeiten von EBC zur Virusdiagnostik, z.B. mittels PCR.

Im klinischen Alltag stehen wir oft vor der Frage, ob bei tiefen Atemwegsinfekten eine antibiotische Behandlung erforderlich ist oder nicht. Hilfreich können hier Biomarker sein, die zwischen bakteriellem und viralem Infekt differenzieren können. Am besten untersucht sind CRP und Procalcitonin (PCT). Während das CRP nur wenig zur Differenzierung zwischen viral und bakteriell beiträgt, scheint das PCT besser geeignet zu sein. Die Stärke des PCT liegt aber eher darin, schwere bakterielle Infekte zu detektieren, als sicher virale Infekte zu erkennen. Ein neuer Marker, der in akuten Infekten möglicherweise hilft, gut zwischen bakteriellen und viralen Infekten zu unterscheiden, ist das Serum Amyloid A. Seine Serumspiegel korrelieren gut mit denen des CRP, weisen aber bei viralen Infektionen bis zu zehnmal höhere Werte auf. Die Fortschritte in der Virusdiagnostik der letzten Jahre haben sehr viel zu unserem Verständnis der Genese der tiefen Atemwegsinfekte beigetragen. Insbesondere gelingt wesentlich häufiger der Nachweis von Viren bei diesen Erkrankungen und es werden neue Viren entdeckt, die eine ätiologische Rolle zu spielen scheinen. Es bleibt jedoch offen zu klären, ob wirklich jeder Virusnachweis auch Krankheitswert hat. Deshalb werden im zweiten Teil dieser Serie die vier häufigsten Formen der tiefen Atemwegsinfekte (akute Bronchitis, akut exazerbierte COPD, Pneumonie und Influenza) vorgestellt und die Bedeutung von Viren bei ihrer Entstehung beleuchtet.

\section{Interessenkonflikte}

$\nabla$

\section{Keine}

\section{Institute}

1 Helios Klinikum Emil von Behring, Zentrum für Pneumologie und Thoraxchirurgie Heckeshorn, Klinik für Pneumologie, Berlin

2 Universitätsklinik und Poliklinik für Pneumologie, Inselspital Bern, und Universität Bern, Bern, Schweiz

3 Berufgenossenschaftliche Universitätsklinik Bergmannsheil, Klinik für PneumologieBochum

${ }^{4}$ Universitätsklinikum Giessen und Marburg, Standort Marburg

5 Medizinische Hochschule Hannover, Klinik für Pneumologie, Hannover

${ }^{6}$ Universitätsklinikum Ulm, Sektion Pneumologie, Klinik für Innere Medizin II, Ulm

Medizinische Universität Wien, Klinik für Innere Medizin I, Wien, Österreich

8 Lehr- und Forschungsgebiet Virologie, Institut für Medizinische Mikrobiologie, Universitätsklinikum Aachen, RWTH Aachen,

9 Bayerisches Landesamt für Gesundheit und Lebensmittelsicherheit, München

\section{Literatur}

1 Office of Population Census and Surveys. Morbidity statistics from general practice, Fourth national study. 1991 -1992, 1995

2 Huchon, G. Management of adult community-acquired lower respiratory tract infections. Eur Respir J 1998; 8: 391 - 426

3 Macfarlane JT, Colville A, Guion A et al. Prospective study of aetiology and outcome of adult lower-respiratory-tract infections in the community. Lancet 1993; 341: $511-514$

4 Macfarlane J, Holmes W, Gard P et al. Prospective study of the incidence, aetiology and outcome of adult lower respiratory tract illness in the community. Thorax 2001; 56: 109-114

5 Fahey T, Stocks N, Thomas T. Quantitative systematic review of randomised controlled trials comparing antibiotic with placebo for acute cough in adults. BMJ 1998; 316: $906-910$

6 Anthonisen NR, Manfreda J, Warren CP et al. Antibiotic therapy in exacerbations of chronic obstructive pulmonary disease. Ann Intern Med 1987; 106: $196-204$

7 Department of Health Standing Medical Advisory Committee Subgroup on Antimicrobial Resistance. The path of least resistance - main report. 2000

8 Ruiz M, Ewig S, Marcos MA et al. Etiology of community-acquired pneumonia: impact of age, comorbidity, and severity. Am J Respir Crit Care Med 1999; 160: 397 - 405 
9 Lim WS, Macfarlane JT, Boswell TC et al. Study of community acquired pneumonia aetiology (SCAPA) in adults admitted to hospital: implications for management guidelines. Thorax 2001; 56: 296-301

10 Höffken G, Lorenz J, Kern W et al. S3-Leitlinie zur Epidemiologie, Diagnostik, antimikrobieller Therapie und Management von erwachsenen Patienten mit ambulant erworbenen tiefen Atemwegsinfektionen (akute Bronchitis, akute Exazerbation einer chronischen Bronchitis, Influenza und anderen respiratorischen Virusinfektionen) sowie ambulant erworbener Pneumonie. Pneumologie 2005; 59: 612 -664

11 Creer DD, Dilworth JP, Gillespie SH et al. Aetiological role of viral and bacterial infections in acute adult lower respiratory tract infection (LRTI) in primary care. Thorax 2006; 61: $75-79$

12 de Roux A, Marcos MA, Garcia E et al. Viral community-acquired pneumonia in nonimmunocompromised adults. Chest 2004; 125: $1343-$ 1351

13 Rohde G, Wiethege A, Borg I et al. Respiratory viruses in exacerbations of chronic obstructive pulmonary disease requiring hospitalisation: a case-control study. Thorax 2003; 58: 37-42

14 Lemaitre B, Nicolas E, Michaut L et al. The dorsoventral regulatory gene cassette spatzle/Toll/cactus controls the potent antifungal response in Drosophila adults. Cell 1996; 86: 973-983

15 Tabeta K, Georgel P, Janssen E et al. Toll-like receptors 9 and 3 as essential components of innate immune defense against mouse cytomegalovirus infection. Proc Natl Acad Sci U S A 2004; 101: 3516- 3521

16 Krug A, French AR, Barchet $W$ et al. TLR9-dependent recognition of MCMV by IPC and DC generates coordinated cytokine responses that activate antiviral NK cell function. Immunity 2004; 21: 107-119

17 Kurt-Jones EA, Popova L, Kwinn L et al. Pattern recognition receptors TLR4 and CD14 mediate response to respiratory syncytial virus. Nat Immunol 2000; 1: $398-401$

18 Hoebe K, Du X, Georgel P et al. Identification of Lps2 as a key transducer of MyD88-independent TIR signalling. Nature 2003; 424: 743-748

19 Jude BA, Pobezinskaya Y, Bishop J et al. Subversion of the innate immune system by a retrovirus. Nat Immunol 2003; 4: 573-578

20 Lund J, Sato A, Akira S et al. Toll-like receptor 9-mediated recognition of Herpes simplex virus-2 by plasmacytoid dendritic cells. J Exp Med 2003: 198: $513-520$

21 Barton GM, Kagan JC, Medzhitov R. Intracellular localization of Toll-like receptor 9 prevents recognition of self DNA but facilitates access to viral DNA. Nat Immunol 2006; 7: 49-56

22 Triantafilou K, Vakakis E, Orthopoulos G et al. TLR8 and TLR7 are involved in the host's immune response to human parechovirus 1 . Eur J Immunol 2005; 35: 2416-2423

23 Crozat $K$;Beutler B. TLR7: A new sensor of viral infection. Proc Natl Acad Sci USA 2004; 101: 6835-6836

24 Kawai T, Sato S, Ishii KJ et al. Interferon-alpha induction through Tolllike receptors involves a direct interaction of IRF7 with MyD88 and TRAF6. Nat Immunol 2004; 5: 1061 - 1068

25 Zhang X, Wang C, Schook LB et al. An RNA helicase, RHIV -1, induced by porcine reproductive and respiratory syndrome virus (PRRSV) is mapped on porcine chromosome 10q13. Microb Pathog 2000; 28: $267-$ 278

26 Pichlmair A, Schulz O, Tan CP et al. RIG-I-mediated antiviral responses to single-stranded RNA bearing 5'-phosphates. Science 2006; 314: 997- 1001

27 Hornung V, Ellegast J, Kim S et al. 5'-Triphosphate RNA is the ligand for RIG-I. Science 2006; 314: 994 - 997

28 Triantafilou K, Orthopoulos G, Vakakis E et al. Human cardiac inflammatory responses triggered by Coxsackie B viruses are mainly Tolllike receptor (TLR) 8-dependent. Cell Microbiol 2005; 7: 1117-1126

29 Honda K, Takaoka A, Taniguchi T. Type I interferon [corrected] gene induction by the interferon regulatory factor family of transcription factors. Immunity 2006; $25: 349-360$

30 Schnare M, Barton GM, Holt AC et al. Toll-like receptors control activation of adaptive immune responses. Nat Immunol 2001; 2: 947-950

31 Andoniou CE, Andrews DM, Degli-Esposti MA. Natural killer cells in viral infection: more than just killers. Immunol Rev 2006; 214: 239-250

32 Klotman ME, Chang TL. Defensins in innate antiviral immunity. Nat Rev Immunol 2006; 6: 447-456

33 Sapey E, Stockley RA. COPD exacerbations. 2: aetiology. Thorax 2006; 61: $250-258$

34 Papi A, Johnston SL. Rhinovirus infection induces expression of its own receptor intercellular adhesion molecule 1 (ICAM-1) via increased NFkappaB-mediated transcription. J Biol Chem 1999; 274: 9707-9720
35 Aaron SD, Angel JB, Lunau $M$ et al. Granulocyte inflammatory markers and airway infection during acute exacerbation of chronic obstructive pulmonary disease. Am J Respir Crit Care Med 2001; 163: 349- 355

36 Qiu Y, Zhu J, Bandi V et al. Biopsy neutrophilia, neutrophil chemokine and receptor gene expression in severe exacerbations of chronic obstructive pulmonary disease. Am J Respir Crit Care Med 2003; 168: 968-975

37 Gompertz S, O'Brien C, Bayley DL et al. Changes in bronchial inflammation during acute exacerbations of chronic bronchitis. Eur Respir ] 2001; $17: 1112-1119$

38 Roland M, Bhowmik A, Sapsford RJ et al. Sputum and plasma endothelin-1 levels in exacerbations of chronic obstructive pulmonary disease. Thorax 2001; 56: 30 - 35

39 Kleines M, Scheithauer S, Rackowitz A et al. High prevalence of human bocavirus detected in young children with severe acute lower respiratory tract disease by use of a standard PCR protocol and a novel realtime PCR protocol. J Clin Microbiol 2007; 45: 1032 - 1034

40 Drosten C, Gunther S, Preiser W et al. Identification of a novel coronavirus in patients with severe acute respiratory syndrome. N Engl J Med 2003; 348: 1967-1976

41 van der Hoek L, Pyrc K, Jebbink MF et al. Identification of a new human coronavirus. Nat Med 2004; 10: 368-373

42 Woo SC, Lau SK, Tsoi HW et al. Clinical and molecular epidemiological features of coronavirus HKU1-associated community-acquired pneumonia. J Infect Dis 2005; 192: 1898 - 1907

43 van den Hoogen BG, de Jong JC, Groen J et al. A newly discovered human pneumovirus isolated from young children with respiratory tract disease. Nat Med 2001; 7: 719-724

44 Principi N, Bosis S, Esposito S. Human metapneumovirus in paediatric patients. Clin Microbiol Infect 2006; 12: 301-308

45 Johnstone J, Majumdar SR, Fox JD et al. Human metapneumovirus pneumonia in adults: results of a prospective study. Clin Infect Dis 2008; 46: $571-574$

46 Cunha BA, Eisentstein LE, Dillard T et al. Herpes simplex virus (HSV) pneumonia in a heart transplant: diagnosis and therapy. Heart Lung 2007; 36: $72-78$

47 Carstens J, Andersen KH, Spencer E et al. Cytomegalovirus infection in renal transplant recipients. Transpl Infect Dis 2006; 8: 203-212

48 Marzouk K, Corate L, Saleh S et al. Epstein-Barr-virus-induced interstitial lung disease. Curr Opin Pulm Med 2005; 11: 456-460

49 Gerna G, Vitulo P, Rovida F et al. Impact of human metapneumovirus and human cytomegalovirus versus other respiratory viruses on the lower respiratory tract infections of lung transplant recipients. J Med Virol 2006; 78: $408-416$

50 Malmstrom K, Pitkaranta A, Carpen $O$ et al. Human rhinovirus in bronchial epithelium of infants with recurrent respiratory symptoms. J Allergy Clin Immunol 2006; 118: 591 - 596

51 Cate TR, Couch RB, Fleet WF et al. Production of Tracheobronchitis in Volunteers with Rhinovirus in a Small-Particle Aerosol. Am J Epidemiol 1965; 81: 95-105

52 Harju TH, Leinonen M, Nokso-Koivisto J et al. Pathogenic bacteria and viruses in induced sputum or pharyngeal secretions of adults with stable asthma. Thorax 2006; 61: 579-584

53 Mosser AG, Vrtis R, Burchell L et al. Quantitative and qualitative analysis of rhinovirus infection in bronchial tissues. Am J Respir Crit Care Med 2005; 171: 645-651

54 Simpson JL, Moric I, Wark PA et al. Use of induced sputum for the diagnosis of influenza and infections in asthma: a comparison of diagnostic techniques. J Clin Virol 2003; 26: 339-346

55 Xiang X, Qui D, Chan KP et al. Comparison of three methods for respiratory virus detection between induced sputum and nasopharyngeal aspirate specimens in acute asthma. J Virol Methods 2002; 101: 127 133

56 Rohde G, Drosten C, Borg I et al. Nachweis von Atemwegsviren - Wie, warum, wann und wo? Pneumologie 2009; 63: 14-22

57 Steininger C, Kundi M, Aberle SW et al. Effectiveness of reverse transcription-PCR, virus isolation, and enzyme-linked immunosorbent assay for diagnosis of influenza $A$ virus infection in different age groups. J Clin Microbiol 2002; 40: 2051-2056

58 Steininger C, Redlberger M, Graninger $W$ et al. Near-patient assays for diagnosis of influenza virus infection in adult patients. Clin Microbiol Infect 2009; 15: $267-273$

59 Kuypers J, Wright N, Ferrenberger, J et al. Comparison of real-time PCR assays with fluorescent-antibody assays for diagnosis of respiratory virus infections in children. J Clin Microbiol 2006; 44: 2382 - 2388 
60 Stroud M, Swindell B, Bernard GR. Cellular and humoral mediators of sepsis syndrome. Crit Care Nurs Clin North Am 1990; 2: 151 - 160

61 Simon L, Gauvin F, Amre DK et al. Serum procalcitonin and C-reactive protein levels as markers of bacterial infection: a systematic review and meta-analysis. Clin Infect Dis 2004; 39: 206 - 217

62 Meisner M, Brunkhorst FM, Reith HB et al. Clinical experiences with a new semi-quantitative solid phase immunoassay for rapid measurement of procalcitonin. Clin Chem Lab Med 2000; 38: 989-995

63 Daubin C, Parienti JJ, Vabret A et al. Procalcitonin levels in acute exacerbation of COPD admitted in ICU: a prospective cohort study. BMC Infect Dis 2008; 8: 145

64 Stolz D, Christ-Crain M, Bingisser R et al. Antibiotic treatment of exacerbations of COPD: a randomized, controlled trial comparing procalcitonin-guidance with standard therapy. Chest 2007; 131: 9-19

65 Christ-Crain M, Stolz D, Bingisser R et al. Procalcitonin guidance of antibiotic therapy in community-acquired pneumonia: a randomized trial. Am J Respir Crit Care Med 2006; 174: 84-93

66 Lannergard , A, Larsson A, Kragsbjerg P et al. Correlations between serum amyloid $A$ protein and $C$-reactive protein in infectious diseases. Scand J Clin Lab Invest 2003; 63: 267-272

67 Steininger C, Graninger W, Zoufaly A et al. Asymptomatic CMV viremia is associated with increased levels of serum amyloid $A$ in patients with advanced HIV-infection. Eur J Med Res 2008; 13: 304 - 308

68 Horvath I, Hunt J, Barnes PJ et al. Exhaled breath condensate: methodological recommendations and unresolved questions. Eur Respir J 2005; 26: $523-548$

69 Majewska E, Kasielski M, Luczynski R et al. Elevated exhalation of hydrogen peroxide and thiobarbituric acid reactive substances in patients with community acquired pneumonia. Respir Med 2004; 98 : $669-676$

70 Corradi M, Pesci A, Casana R et al. Nitrate in exhaled breath condensate of patients with different airway diseases. Nitric Oxide 2003; 8: 26-30

71 Vaughan J, Ngamtrakulpanit L, Pajewski TN et al. Exhaled breath condensate $\mathrm{pH}$ is a robust and reproducible assay of airway acidity. Eur Respir J 2003; 22: 889-894

72 Kostikas K, Papatheodorou G, Ganas K et al. pH in expired breath condensate of patients with inflammatory airway diseases. Am J Respir Crit Care Med 2002; 165: $1364-1370$

73 Hunt JF, Fang $K$, Malik $R$ et al. Endogenous airway acidification. Implications for asthma pathophysiology. Am J Respir Crit Care Med 2000; 161: $694-699$
74 Tate S, MacGregor G, Davis $M$ et al. Airways in cystic fibrosis are acidified: detection by exhaled breath condensate. Thorax 2002; 57: $926-$ 929

75 Gessner C, Hammerschmidt S, Kuhn H et al. Exhaled breath condensate acidification in acute lung injury. Respir Med 2003; 97: 1188-1194

76 Suliman HB, Ryan LK, Bishop L et al. Prevention of influenza-induced lung injury in mice overexpressing extracellular superoxide dismutase. Am J Physiol Lung Cell Mol Physiol 2001; 280: L69-78

77 Carpagnano GE, Foschino-Barbaro MP, Mule G et al. 3p microsatellite alterations in exhaled breath condensate from patients with non-small cell lung cancer. Am J Respir Crit Care Med 2005; 172: 738 - 744

78 Gessner C, Kuhn H, Toepfe K et al. Detection of p53 gene mutations in exhaled breath condensate of non-small cell lung cancer patients. Lung Cancer 2004; 43: 215-222

79 Agoritsas K, Mack K, Bonsu BK et al. Evaluation of the Quidel QuickVue test for detection of influenza $A$ and $B$ viruses in the pediatric emergency medicine setting by use of three specimen collection methods. J Clin Microbiol 2006; 44: 2638-2641

80 Grijalva CG, Poehling KA, Edwards KM et al. Accuracy and interpretation of rapid influenza tests in children. Pediatrics 2007; 119: e6-11

81 Carraro E, Neto DF, Benfica $D$ et al. Applications of a duplex reverse transcription polymerase chain reaction and direct immunofluorescence assay in comparison with virus isolation for detection of influenza A and B. Diagn Microbiol Infect Dis 2007; 57: 53-57

82 Shetty AK, Treynor E, Hill DW et al. Comparison of conventional viral cultures with direct fluorescent antibody stains for diagnosis of community-acquired respiratory virus infections in hospitalized children. Pediatr Infect Dis J 2003; 22: 789-794

83 Vareckova E, Blaskovicoa H, Gocnik M et al. Evaluation of clinical specimens for influenza A virus positivity using various diagnostic methods. Acta Virol 2006; 50: 181 - 186

\section{Bisher erschienene Beiträge dieser Serie \\ $\nabla$}

1 Strassburg A et al. Infektionsdiagnostik in der Pneumologie. Pneumologie 2008; 62: 730-743

2 Rohde $G$ et al. Nachweis von Atemwegsviren - wie, warum, wann und wo? Pneumologie 2009; 63: 14-22

3 Gillissen A et al. Biomarker bei infektiösen und nicht infektiösen Lungenerkrankungen außer Malignome. Pneumologie 2009; 63: 439-450 